\title{
Topological thermal instability and the length of proteins
}

\author{
Raffaella Burioni and Davide Cassi \\ Dipartimento di Fisica and INFM, Università di Parma, \\ Parco Area delle Scienze 7A, 43100 Parma, Italy \\ Fabio Cecconi and Angelo Vulpiani \\ Dipartimento di Fisica and INFM (UdR and SMC), \\ Università di Roma La Sapienza Piazzale A. Moro 2, 00185 Roma, Italy
}

\begin{abstract}
We present an analysis of the role of global topology on the structural stability of folded proteins in thermal equilibrium with a heat bath. For a large class of single domain proteins, we compute the harmonic spectrum within the Gaussian Network Model (GNM) and we determine the spectral dimension, a parameter describing the low frequency behaviour of the density of modes. We find a surprisingly strong correlation between the spectral dimension and the number of aminoacids of the protein. Considering that the larger the spectral dimension, the more topologically compact is the folded state, our results indicate that for a given temperature and length of the protein, the folded structure corresponds to the less compact folding compatible with thermodynamic stability.
\end{abstract}

\section{INTRODUCTION}

The role of geometry has recently been considered as a factor of primary importance for the comprehension of several physical properties of proteins and other biological macromolecules. In particular, since the topology of folded states is known to influence the folding properties [1, 2, 3, 4, 5, 6, 7, 8] , a great deal of work has been devoted to the study of topological aspects describing the networks of links between aminoacids in folded proteins. 9, 10, 11]. Futhermore, relevant features of protein conformations seem to follow the geometrical principles of the optimal packing problem [12, 13].

Starting from the primary, linear, structure (the sequence of aminoacids), a protein evolves during the folding process until it reaches a final state (native state) whose geometrical shape is crucial to the function of the protein itself. However, the problem of the topological arrangement of proteins in their native states cannot be regarded as purely static issue. Indeed, a massive accumulation of experimental data collected from X-ray, NMR and neutron spectroscopy, has reveiled that protein native states are rather dynamic structures where aminoacids constantly move around equilibrium positions. This dynamics, crucially involved in protein functions [14, 15], is usually examined and investigated through normal modes analysis (NMA) [16] and its variants [17].

The study of collective motions of large proteins is generally of difficult accessibility to realistic all-atoms NMA [8] and simplified or approximate approaches are usually welcome. Tirion 19 first proposed the possibility to replace, in protein normal mode computations, complicated empirical potentials by Hookian pairwise interactions depending on a single parameter. The approach stems from the observation that low-frequency dynamics, which is mainly associated with protein-domain motion, is generally insensitive to the finer details of atomic interactions. A vast further literature 20, 21, 22, 23] confirmed the success of simple harmonic models in the study of slow vibrational dynamics of large biological macromolecules, until they become a viable alternative to heavy and time-consuming all-atoms NMA. This success relies on the striking agreement with experiments, on the presence of few adjustable parameters and finally on their easy numerical implementation on computers and fast result production. For such reasons harmonic models are suitable also for the systematic analysis of large datasets of proteins.

The topological stability of macromolecules is far from being a pure mechanical problem as it closely involves thermodynamics: indeed, the relevant thermodynamic potential to minimize in order to find the stable configuration is not an energy, but a free energy. This is due to the interaction with the environment (schematized as a thermal bath) which is generally not negligible, specifically for biological macromolecules having a stable phase in a solvent. In particular, water is a very efficient medium for the transfer of thermal energy at microscopic scales (i.e. oscillations and molecular rotations).

Following these considerations, in this work we apply the approach of NMA to investigate the influence of the global native state topology on the thermal stability of proteins.

Vibrational thermal instability is a well-known issue in solid state physics. Since the first classical analysis of Peierls [24], it has been recognized that the equilibrium with a thermal bath can dramatically influence the allowed topological arrangement of large geometrical structures. The most striking consequence of Peierls instability has concerned up to now low-dimensional crystals: for one and two-dimensional lattices the mean square displacement of 
a single atom at finite temperature diverges in the thermodynamic limit, i.e. with increasing number of atoms. When the displacement exceeds the order of magnitude of the lattice spacing, the topological arrangement of the lattice is unstable and the crystal becomes a liquid. For real structures, formed by a finite number of units and far from the thermodynamic limit, the divergence sets a maximal stability size, which is negligible for one-dimensional lattices and typically mesoscopic for two-dimensional lattices.

However, thermal instability is present not only in crystals but also in the case of structurally inhomogeneous systems, such as, glasses, fractals, polymers and non crystalline structures. There, the problem is much more complex. Generalizing the Peierls approach to mesoscopic disordered structures, we are able to apply this kind of arguments to the thermal stability of macromolecules. In the following we describe how this can be done in the case of proteins; we will predict the existence of a critical stability size depending on a global topological parameter (the spectral dimension) and compare our predictions with experimental data.

\section{THEORY}

In a recent paper [25] generalizing the Peierls' result, we have shown that a thermodynamic instability appears also in inhomogeneous structures and it is determined by the spectral dimension $\bar{d}$. The parameter $\bar{d}[26]$ is defined according to the asymptotic behaviour of the density of harmonic oscillations at low frequencies. More precisely, denoting by $\rho(\omega)$ the density of modes with frequency $\omega$, then

$$
\rho(\omega) \sim \omega^{\bar{d}-1}
$$

for $\omega \rightarrow 0$. The spectral dimension is the most natural extension of the usual Euclidean dimension $d$ to disordered structures as far as dynamical processes are concerned. It coincides with $d$ in the case of lattices, but in general, it can assume non-integer values between 1 and 3. The spectral dimension represents a useful measure of the effective connectedness of geometrical structures at large scales, because large values of $\bar{d}$ correspond to high topological connectedness. Moreover, it characterizes not only harmonic oscillations, but it is also related to diffusion, phase transitions and electrical conductivity, allowing a variety of both experimental and numerical methods for its determination [27, 28]. The relevance of $\bar{d}$ in connection with the anomalous density of vibrational modes in proteins has also been considered in refs. 229, 30].

In the case of thermal instability, we demostrated that, for $\bar{d} \geq 2$, the mean square displacement $\left\langle r^{2}\right\rangle$ of a structural unit (being an atom, a molecule or a supramolecular structure according to the studied case) of a system composed of $N$ elements, diverges in the limit $N \rightarrow \infty$. Denoting with $T$ the temperature of the heat bath and with $K_{B}$ the Boltzmann constant, the divergence is given by the asymptotic law:

$$
\left\langle r^{2}\right\rangle \sim K_{B} T N^{2 / \bar{d}-1}
$$

for $\bar{d}<2$. For $\bar{d}=2$, the mean square displacement diverges logarithmically, $\left\langle r^{2}\right\rangle \sim K_{B} T \ln N$, as in the case of the Peierls' result for a two dimensional crystal. Notice that the divergence in $\left\langle r^{2}\right\rangle$ is only determined by $\bar{d}$. Now, at any given temperature $T$, there will exist a threshold value $N(T)$ beyond which $\left\langle r^{2}\right\rangle^{1 / 2}$ exceeds the typical spacing between nearest neighbors, making the solid structure unstable. Therefore at large enough $N$, the solid will experience a structural reorganization which can lead either to a homogeneous liquid phase at sufficiently high temperatures or to a disordered 3-dimensional solid, which is homogeneous at large scales and inhomogeneous at small scales. In general, the threshold values of $N$ are very small with respect to the typical order of magnitude of macroscopic systems, being rather comparable to the size of large complex macromolecules such as biopolymers.

This poses an intriguing question concerning proteins. Indeed, to exploit their biological function proteins must keep a specified geometrical and topological arrangement and cannot afford any, even partial, geometrical large scale fluctuations as it happens, instead, to swallen polymeric chains in a good solvent 31]. This makes thermodynamical stability problems of crucial importance and suggests a possible correlation between the spectral dimension and the length of protein chains.

The vibrational stability problem in proteins has been analysed through the gaussian network model (GNM), proposed in [19], which yields results in agreement with principal X-ray spectroscopy experiments. This approach generally considers proteins as elastic networks, whose nodes are the positions of the alpha-carbons $\left(\mathrm{C}_{\alpha}\right)$ in the native structure and the interactions between nodes are assimilated to harmonic springs. The only information required to implement the method is the knowledge of the native structure, and only two free parameters are introduced, the 
spring constant and interaction cutoff. The GNM is defined by the quadratic Hamiltonian

$$
H=\sum_{i}^{N} \frac{\mathbf{p}_{i}^{2}}{2 m}+\frac{\gamma}{2} \sum_{i j} \Delta_{i j}\left(\delta \mathbf{r}_{i}-\delta \mathbf{r}_{j}\right)^{2}
$$

where the first term is the kinetic energy of the system, $\gamma$ being the strength of the springs that are assumed homogeneous, $\mathbf{R}_{i}$ and $\delta \mathbf{r}_{i}$ indicating the equilibrium position and the displacement with respect to $\mathbf{R}_{i}$ of the $i$-th $\mathrm{C}_{\alpha}$ atoms. The model is eventually defined by the contact matrix $\Delta$ with entries: $\Delta_{i j}=1$ if the distance $\left|\mathbf{R}_{i}-\mathbf{R}_{j}\right|$ between two $\mathrm{C}_{\alpha}$ 's, in the native conformation, is below the cutoff $R_{0}=0.6 \AA$, while is 0 otherwise.

The harmonic spectrum for each structure is given by the set of eigenvalues $\left\{\omega_{1}, \ldots, \omega_{N}\right\}$ of the Kirchhoff matrix (or valency-adjaciency matrix) $\Gamma_{i j}=-\Delta_{i j}+\delta_{i j} \sum_{l \neq i} \Delta_{i l}$,

Notice that the first eigenvalue $\omega_{1}$ vanishes and corresponds to the constant eigenvector, describing the trivial uniform translation.

The comparison between experimental data and GNM results is obtained via the X-ray crystallographyc B-factors, measuring the mean square fluctuation of $\mathrm{C}_{\alpha}$ atoms around native positions

$$
B_{i}(T)=\frac{8 \pi^{2}}{3}\left\langle\delta \mathbf{r}_{i} \delta \mathbf{r}_{i}\right\rangle
$$

In the GNM approximation, these can be expressed in terms of the diagonal part of the inverse of $\Gamma$ [32]:

$$
\left\langle\delta \mathbf{r}_{i} \delta \mathbf{r}_{j}\right\rangle=\frac{3 K_{B} T}{\gamma}\left[\Gamma^{-1}\right]_{i j}
$$

The comparison with crystallographyc data is crucial for setting the correct values of the free parameters $R_{0}$ end $\gamma$ of the GNM (see. Methods and Results).

\section{METHODS AND RESULTS}

We present now the harmonic analysis of the GNM performed over the dataset of protein native structures with different sizes listed in Table 1. and downloaded from the Brookheaven Protein Data Bank. We selected our representative statistical sample according to the following criteria: first, we only considered proteins with a stable large scale geometry. This excludes multiple domains proteins, where the domains can move independently giving rise to large geometrical fluctuations. Moreover, we selected proteins not binded to fragments of DNA, RNA or other substrates because such structures cannot be described with sufficient accuracy in terms of simple harmonic model with only two effective parameters.

Finally, we choose proteins covering uniformly a wide length interval ranging from 100 to 3600 to test our prediction.

The diagonalization of the Kirchhoff matrix $\Gamma$ to obtain its eigenvalues $\left\{\omega_{1}^{2}, \ldots, \omega_{n}^{2}\right\}$ and eigenvectors has been performed with the standard numerical packages [33]. The knowledge of the eigenvectors (eigenmodes) allows to compute the GNM B-factors:

$$
B_{i}(T)=\frac{8 \pi^{2} K_{B} T}{\gamma} \sum_{k} \frac{\left|u_{i}(k)\right|^{2}}{\omega_{k}^{2}}
$$

where $i$ is the residue index, the sum runs over all non-zero frequencies and $\mathbf{u}(\mathbf{k})_{i}$ indicates the $i$-th component of the $k$-th eigenmode.

When generating the contact matrix $\Delta$, the value of the interaction cutoff $R_{0}$ has been chosen in order to have agreement between the experimental and the GNM B-factors for each proteins, as showed in Fig. 11. We found that the average value $R_{0}=6 \AA$ was a consistent choice for the whole set of proteins.

The purpose of our analysis is basically:

a) investigating whether there exists a correlation between the spectral dimension of native structures and the length of natural occurring proteins;

b) in the case of positive result for point a), verifying whether the correlation is connected with equation (2). 
This expression establishes a rather strong relation between the spectral dimension and the maximum size $N_{\text {max }}$ of a protein. Since the stability is supposed to fail when the fluctuation $\left\langle r^{2}\right\rangle^{1 / 2}$ becomes of the same order of magnitude of the mean distance between non consecutive aminoacids ( $6 \AA$ ), one has

$$
\frac{1}{\ln \left(N_{\max }\right)}=a(2 / \bar{d}-1)
$$

The proportionality constant $a$ in (4) depends on the mean aminoacid spacing, on the spring elastic constant g and temperature T. However, this dependece is very weak (i.e.only logarithmic). This allows for a comparison of different proteins without the computation of the specific parameters.

Actually, equation (4), being based uniquely on thermodynamics stability, can be regarded as an upper bound prediction only.

In Figure 2, we plot the harmonic spectrum, obtained within the GNM, for three proteins with sizes, small, medium and large, respectively. The low frequency regions clearly exhibit a power law behavior whose exponent $\beta$ is directly related to the spectral dimension via formula $\bar{d}=2 / \beta$.

Figure 3 summarises the results of our analysis. We plot the $1 / \ln (N)$ versus the computed slope from the spectra. The choice of these variables is suggested by relation (4): indeed, if eqn.(3) holds, we should obtain a straight line crossing the origin.

As matter of fact, our data are very well fitted by a straight line, with linear regression coefficient 0.76 , but described by the equation

$$
\frac{1}{\ln (N)}=a(2 / \bar{d}-1)+b
$$

with parameters $a=0.133$ and $b=1.134$.

\section{DISCUSSION AND CONCLUSIONS}

The neat result expressed by (5) deserves several comments. First, this equation is in agreement with the upper bound we obtained Eq. 4 supporting the relevance of topological thermal instability in constraining the protein geometry. More important, not only the upper bound is satisfied, but the experimental points lie on a straight line parallel to the upper bound one Eq. (4). This suggests a more fundamental role of topological stability: in some sense, the protein tends to arrange topologically in such a way to reach the minimum value compatible with stability constraints. In other words, for any fixed length, it tends to the most swollen state which remains stable with respect to thermal fluctuations.

A very interesting point is the meaning of the parameter $b$ which is 0 in Eq. (4). Its positive value could have different motivations, but its universal nature (it is a "protein-independent" global shift) must be due to a very general mechanism. A rather obvious reason is the contribution of anharmonic interactions at finite temperatures; a more intriguing one could be an effective longer range interaction due to the presence of bound water molecules around the external aminoacids, which could change the effective form of the interaction matrix $\Lambda$. This hypothesis is also suggested by the physical interpretation of $b$ as an anomalous dimension exponent, typically related to a renormalized interaction 34$]$

However, the most intriguing evidence relies on the regression coefficient Independently of the physical origin of $b$, its high value strongly supports the existence of a thermodynamic stability threshold, dependent on the topology of the folded state, for the size of proteins.

[1] Gø N. and Scheraga H.A. On the Use of Classical Statistical Mechanics in the Treatment of Polymer Chain Conformation. Macromolecules 1976;9; 535-549.

[2] Plaxco K.W. ,Simons K.T. and Baker D. Contact order, transition state placement and the refolding rates of single domain proteins. J. Mol. Biol. 1999;277; 985-994.

[3] Makarov D.E., Keller C.A., Plaxco K.W., Metiu H. How the folding rate constant of simple, single-domain proteins depends on the number of native contacts. Proc. Natl. Acad. Sci. USA, 2002;99; 3535-3539.

[4] Riddle D.S., Grantcharova V.P., Santiago J.V., Alm E., Ruczinski I. and Baker D. Experiment and theory highlight role of native state topology in SH3 folding. Nature Struct. Biol. 1999;6; 1016-1024. 
[5] Baker D. A surprising simplicity to protein folding. Nature 2000;405; 39-42.

[6] Clementi C., Nymeyer H. and Onuchic J.N. Topological and energetic factors: What determines the structural details of the transition state ensemble and "en-route" intermediates for protein folding? An investigation for small globular proteins. J. Mol. Biol. 1998;298; 937-953.

[7] Klimov D.K., and Thirumalai D. Native topology determines force-induced unfolding pathways in globular proteins. Proc. Natl. Acad. Sci. USA 2000;97; 7254-7259.

[8] Cecconi F., Micheletti C., Carloni P. and Maritan A. Molecular dynamics studies on HIV-1 protease drug resistance and folding pathways. Proteins: Struc. Func. Gen. 2001;43; 365-372.

[9] Kabakcioglu A., Kanter I., Vendruscolo M. and Domany E. Statistical properties of contact vectors. Phys. Rev. E 2002;65; 041904.

[10] Park K., Vendruscolo M. and Domany E. Toward an energy function for the contact map representation of proteins. Proteins: Struct. Func. Gen. 2000;40; 237-248.

[11] Vendruscolo M., Dokholyan N.V., Paci E. and Karplus M. Small-world view of the amino acids that play a key role in protein folding Phys. Rev. E 2002;65; 061910.

[12] Maritan A., Micheletti C., Trovato A. and Banavar J.R., Optimal shapes of compact strings. Nature 2000;406; 287-290.

[13] Banavar J.R. and Maritan A. Geometrical approach to protein folding: a tube picture. Rev. Mod. Phys. 2003;75; 23-34.

[14] Frauenfelder H., Petsko G.A. and Tsernoglou D. Temperature dependent x-ray diffraction as a probe as of protein structural dynamics. Nature 1979;280; 558-563.

[15] Frauenfelder H., McMahon B. Dynamics and functions of proteins: the search of general concepts. Proc. Natl. Acad. Sci. USA $1998 ; 95 ; 4795-4797$.

[16] Karplus M. and McCammon J. Dynamics of Proteins: Elements and Functions. Ann. Rev. Biochem. 1983;53; 263-300.

[17] Amadei A., Linssen A.B.M., Berendsen H.J.C., Essential dynamics of proteins. Proteins: Struct. Func. Gen. 1993;17; 412-425.

[18] Levitt M., Sanders C. and Stern P.S. Protein normal-mode dynamics; trypsin inhibitor, crambin, ribonuclease, and lysozyme. J. Mol. Biol. $1985 ; 181 ; 423-447$.

[19] Tirion M.M. Low-amplitude elastic motions in proteins from a single-parameter atomic analysis. Phys. Rev. Lett. 1996;77; 1905-1908.

[20] Hinsen K. Analysis of domain motion by approximate normal mode calculations. Proteins: Struct. Funct. Genetics 1999;33; 417-429.

[21] Bahar I., Atilgan A.R., Demirel M.C and Erman B. Vibrational dynamics of folded proteins: Significance of slow and fast motions in relation to function and stability. Phys. Rev. Lett. 1998;80; 2733-2736.

[22] Atilgan A.R., Durell S.R., Jernigan R.L., Demirel M.C., Keskin O. and Bahar I. Anisotropy of fluctuation dynamics of proteins with an elastic network model. Biophys. J. 2001;80; 505-515.

[23] Haliloglu T., Bahar I., and Erman. B. Gaussian dynamics of folded proteins. Phys. Rev. Lett.1997;79; 3090-3093.

[24] Peierls R.E. Bemerkung über Umwandlungstemperaturen. Helv. Phys. Acta 1934;7; S81-S83.

[25] Burioni R., Cassi D., Fontana M.P. and Vulpiani A. Vibrational thermodynamic instability of recursive networks. Europhysics Lett. 2002;58; 806-810.

[26] Alexander S. and Orbach R.L. Density of states on fractals: fractons. J. Phys. Lett. 1982;43; L625-L631.

[27] Burioni R. and Cassi D. Universal properties of spectral dimension. Phys. Rev. Lett. 1996;76; $1091-1093$.

[28] Saviot L., Duval E., Surotsev N., Jal J.F., Dianoux A.J. Propagating to nonpropagating vibrational modes in amorphous polycarbonate. Phys. Rev. B. 1999;60; 18-21.

[29] Ben-Avraham D. Vibrational normal-mode spectrum of globular proteins. Phys. Rev. B 1993;47; 14559.

[30] Elber R. and Karplus M. Low frequency modes in proteins:use of effective-medium approximation to interpret fractal dimension observed in electron-spin relaxation meaurements. Phys. Rev. Lett. 1986;56; 394-397.

[31] De Gennes P.G. Scaling concepts in polymer physics. Cornell University Press, Ithaca (1979).

[32] Bahar I., and Atilgan A.R. and Erman B. Direct evaluation of thermal fluctuations in proteins using a single-parameter harmonic potential. Fold. Des. 1997;2; 173-181.

[33] Press W.H., Flannery B.P., Teukolsky S.A., and Vetterling W.T. Numerical recipes. Cambridge University Press; Cambridge 1993

[34] Goldenfeld N. Lectures on Phase Transitions and the Renormalization Group. Frontiers in Physics 85 Addison-Wesley Publishing Company 1992. 


\section{Bifactor 1A55 Protein}

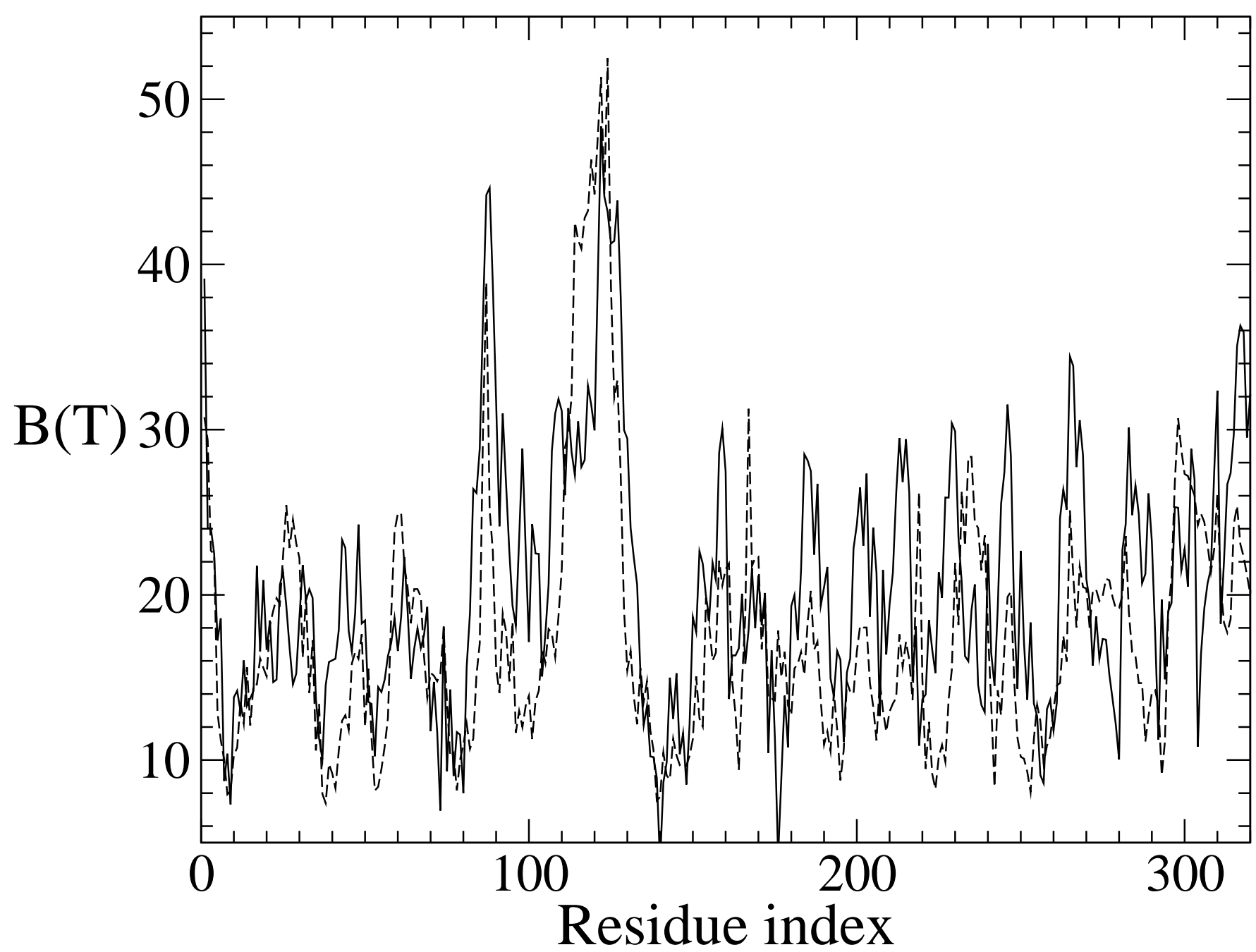

FIG. 1: Comparison of experimental B-factors and mean square fluctuations of Ca yielded by GNM with a cutoff $R_{0}=6 \stackrel{\AA}{\circ}$ for the protein $1 A 55$ with 321 residues. Solid line refers to crystallographic data, while dashed refers to GNM approximation. Since, there is a reasonable overlap between flexible regions of analysed proteins predicted by GNM and experimental indications, this is a good check for the correctness of the cutoff choiche. 


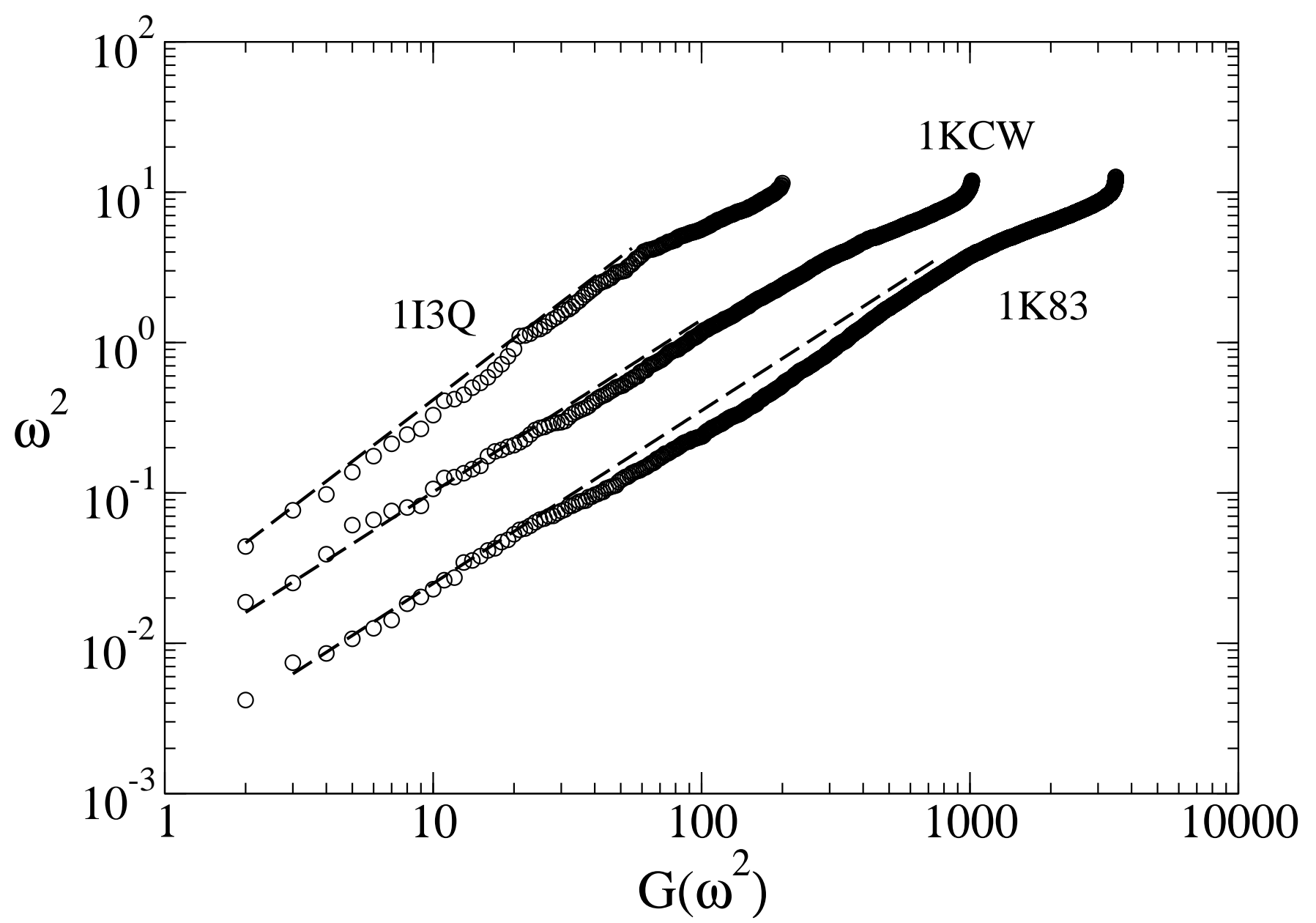

FIG. 2: Log-log plot of the harmonic spectrum from GNM for three proteins of different sizes, 1I3Q $(\mathrm{N}=200), 1 \mathrm{KCW}(\mathrm{N}=1017)$ and $1 \mathrm{~K} 83(\mathrm{~N}=3494)$. On vertical axis, we report the eigenvalues $\omega^{2}$ of the matrix $\Gamma$, and on the orizontal, the number of eigenvalues below a given value $\omega^{2}$, i.e. the integrated density of modes. Dashed lines are the best-fits, whose slope $\beta$, is related to the spectral dimension, $\beta=2 / \bar{d}$ 


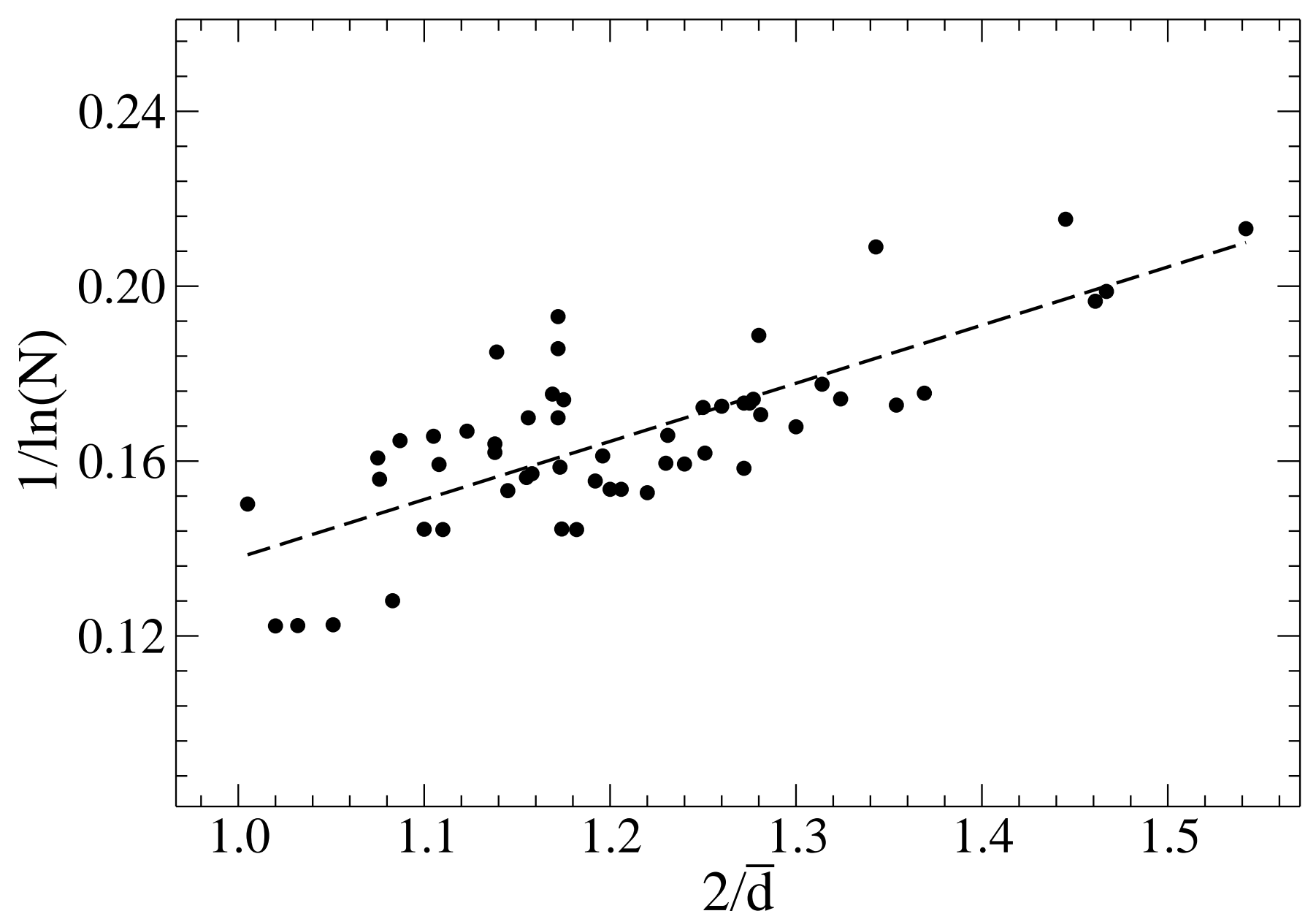

FIG. 3: Linear plot showing the dependence of the spectral dimension on protein size. The line, indicating the behaviour (4), is a best fit with a correlation coefficient 0.76 . 


\begin{tabular}{|c|c|c|c|c|c|}
\hline PDB code & Length & $\bar{d}$ & PDB code & Length & $\bar{d}$ \\
\hline 9RNT & \begin{tabular}{l|l}
104 \\
\end{tabular} & 1.38 & 1DY4 & \begin{tabular}{|l|}
434 \\
\end{tabular} & 1.84 \\
\hline $1 \mathrm{THO}$ & 109 & 1.30 & 1BU8 & 446 & 1.76 \\
\hline 1CCR & 111 & 1.49 & $1 \mathrm{AUK}$ & 480 & 1.76 \\
\hline $1 \mathrm{BVC}$ & 153 & 1.36 & $1 \mathrm{AC} 5$ & 483 & 1.60 \\
\hline 110L & 162 & 1.37 & $1 \mathrm{CPU}$ & 495 & 1.67 \\
\hline $1 \mathrm{G} 12$ & 167 & 1.61 & $1 \mathrm{~A} 65$ & 504 & 1.86 \\
\hline $1 \mathrm{AMM}$ & 174 & 1.55 & $1 \mathrm{SOM}$ & 528 & 1.63 \\
\hline 1IQQ & 200 & 1.57 & 1E3Q & 532 & 1.61 \\
\hline $1 \mathrm{~B} 0 \mathrm{~F}$ & 218 & 1.67 & $1 \mathrm{CRL}$ & 534 & 1.81 \\
\hline 1AE5 & 223 & 1.66 & $1 \mathrm{AKN}$ & 547 & 1.71 \\
\hline $1 \mathrm{~A} 06$ & 279 & 1.52 & 1DIY & 553 & 1.58 \\
\hline $1 \mathrm{~A} 48$ & 298 & 1.46 & $1 \mathrm{CF} 3$ & 582 & 1.73 \\
\hline $1 \mathrm{~A} 3 \mathrm{H}$ & 300 & 1.71 & 1EX1 & 602 & 1.73 \\
\hline $16 \mathrm{VP}$ & 311 & 1.51 & 1A14 & 612 & 1.86 \\
\hline $1 \mathrm{~A} 5 \mathrm{Z}$ & 312 & 1.57 & $1 \mathrm{MZ5}$ & 622 & 1.68 \\
\hline $1 \mathrm{~A} 1 \mathrm{~S}$ & 313 & 1.70 & 1CB8 & 674 & 1.66 \\
\hline $1 \mathrm{~A} 40$ & 321 & 1.57 & $1 \mathrm{HMU}$ & 674 & 1.67 \\
\hline $1 \mathrm{~A} 54$ & 321 & 1.57 & $1 \mathrm{~A} 47$ & 683 & 1.75 \\
\hline $137 \mathrm{~L}$ & 326 & 1.48 & $1 \mathrm{DMT}$ & 696 & 1.64 \\
\hline $1 \mathrm{~A} 20$ & 329 & 1.59 & $1 \mathrm{~A} 4 \mathrm{G}$ & 780 & 1.99 \\
\hline 1A0I & 332 & 1.60 & $1 \mathrm{HTY}$ & 1014 & 1.70 \\
\hline $1 \mathrm{~A} 26$ & 351 & 1.56 & $1 \mathrm{KCW}$ & 1017 & 1.82 \\
\hline 8JDW & 360 & 1.71 & APP1 & 1021 & 1.69 \\
\hline 1BVW & 360 & 1.73 & 1B0P & 2462 & 1.80 \\
\hline 7ODC & 387 & 1.54 & $1 \mathrm{KEK}$ & 2462 & 1.94 \\
\hline 1A39 & 401 & 1.78 & $1 \mathrm{~K} 83$ & 3494 & 1.90 \\
\hline $16 \mathrm{PK}$ & 415 & 1.63 & 1I3Q & 3542 & 1.94 \\
\hline $1 \mathrm{~A} 2 \mathrm{~N}$ & 418 & $1.81 \|$ & $1 \mathrm{I} 50$ & 3558 & 1.96 \\
\hline
\end{tabular}

TABLE I: List of processed native protein structures from Brookheaven PDB, with their lenght and correspondig spectral dimension estimated by GNM approach. 